\title{
Del instrumentalismo a las configuraciones didácticas: una nueva mirada hacia la disciplina
}

\author{
Diana Patricia Landazábal Cuervo ${ }^{1}$ \\ Eliécer Pineda Ballesteros ${ }^{2}$
}

\begin{abstract}
Resumen
La didáctica ha sido considerada por muchos investigadores y docentes como una disciplina de poco valor. Esta tendencia tiene relación con el hecho de considerarla simplemente como método o instrumento. Sin embargo, también algunos investigadores han reflexionado en torno al problema de la enseñanza y del aprendizaje en aula y han venido generando nuevas propuestas. En este artículo se presentan estas reflexiones desde las propuestas iniciales como la de Comenio, hasta el hecho de mirar las configuraciones didácticas como una forma de estudiar y sistematizar el conocimiento didáctico. Se espera que estas reflexiones de los autores del presente artículo, al tomar como base las experiencias de docentes e investigadores, sean propicias para generar nuevas miradas en los lectores. Esta reflexión invita a investigar sobre las prácticas de enseñanza en sus contextos, de forma tal que la didáctica sea además una disciplina para la intervención social.
\end{abstract}

Palabras clave: didáctica, prácticas de enseñanza, intervención social, método, evaluación, configuraciones didácticas. 


\title{
From instrumentalism to educational settings: a new look to the discipline
}

\begin{abstract}
Teaching has been considered by many researchers and teachers as a subject of little value. This trend is related to the fact of considering it simply as a method or instrument. However, some researchers have also thought about the problem of teaching and learning in the classroom and have been generating new proposals. This article presents such reflections from the initial proposals of Comenius, to watching educational settings as a way to study and systematize knowledge for teaching. It is hoped that these reflections of this article's authors, based on teachers and researchers, will be useful for generating new perspectives on readers. This reflection invites to researching on instructional practices in their contexts, so that teaching is also a discipline for social intervention.
\end{abstract}

Key words: didactics, teaching practices, social action, method, evaluation, educational settings.

\section{lntroducción}

La didáctica ha sido considerada a través de los años como una seudociencia o seudodisciplina que tiene poca importancia en las prácticas pedagógicas y de enseñanza. Esta mirada reduccionista de la didáctica, derivada de su definición como un método para aplicar en el aula de clase, ha venido cambiando y se ha empezado a reconocer su importancia como parte del proceso pedagógico.

En este artículo se plantea en primera instancia la necesidad de rescatar el lugar de esta disciplina. En el desarrollo del artículo se presentan algunas consideraciones sobre su objeto de estudio, la didáctica universitaria y algunos problemas que le conciernen, como el método y la evaluación. Seguidamente se hace una revisión de la propuesta de Litwin (1997) sobre el estudio de las configuraciones didácticas y para finalizar se presentan conclusiones de este discernimiento a partir de los diferentes autores. 


\section{Planteamiento de la cuestión}

Mirar hacia un aula de clase es enfrentarse con un conjunto de situaciones que evidencian una serie de procesos que allí se producen; donde sus protagonistas, maestro y estudiantes, configuran una relación que ante todo es humana y que por lo tanto es compleja y particular. El espacio del aula de clase se constituye en el lugar donde la enseñanza y el aprendizaje se conjugan en un espacio social y comunicativo, reflejo de construcción y negociación de significaciones que permiten la subjetivización del alumno y del profesor, donde la cultura y el contexto político median y ejercen tensiones que influyen en las "formas" y los "estilos" de relacionarse de cada uno.

En el aula, el profesor y sus alumnos asumen posturas frente a la construcción de conocimiento y su proceso formativo, por lo cual cada espacio debe comprenderse asumiendo su particularidad, ya que corresponde a una época, responde a un pensamiento y está demarcado por las políticas educativas e institucionales, y aspectos sociales y culturales en los que se configuran los "modos" de actuar de los docentes y del estudiante.

Por tanto, observar lo que acontece en un salón de clase universitaria es apasionante para aquel que considera este espacio como un lugar de investigación que permite explorar las complejas relaciones que se dan, primero, entre los agentes educativos en torno a: (a) diferentes objetivos (formativos, instruccionales, profesionalizantes, etc.); (b) la enseñanza y formación profesional del individuo; (c) los intereses particulares de los docentes y los institucionales; (d) la enseñanza como práctica intencionada y el aprendizaje, y finalmente, (e) educación, enseñanza y cultura.

El aula puede verse como un sistema complejo de investigación y construcción de conocimiento. El aula "aun siendo más que la suma de las partes (profesores y alumnos individualmente hablando), no es cognoscible sino conociendo también las partes". Es abierta y de naturaleza social y epistemológica, permite el flujo permanente de información y es un sistema de comunicación formado por una red de emisores, receptores y canales (Porlan et al., 1998).

Ante esto surge una pregunta. ¿Cuál es la ciencia que puede explicar los procesos complejos y las relaciones que se dan en el aula? Algunos han considerado que esta ciencia es precisamente la didáctica, porque es una disciplina que teoriza sobre las prácticas de enseñanza, aportando elementos que son fundamentales para generar mejores prácticas de enseñanza y que en un sentido más amplio permite comprenderlas desde diferentes dimensiones. "La didáctica es una teoría de la enseñanza, heredera y deudora de muchas otras disciplinas. Su destino, empero, al ocuparse de la acción pedagógica es constituirse recíprocamente en oferente y dadora de teoría en el campo de la acción social y del conocimiento" (De Camilloni et al., 1998). 
Por otra parte, al conformarse la didáctica como teoría, es orientadora de las prácticas de enseñanza al ser el conjunto de conocimiento del que parte el profesor para intervenir sobre el grupo. Así, la didáctica es ante todo una disciplina de intervención social, porque desarrolla estrategias que favorecen la formación humana, la autonomía y la construcción del conocimiento.

Este artículo, producto de una revisión, realiza un análisis desde la historia de la didáctica hasta los problemas actuales que enfrenta la disciplina como el método y la evaluación. Al reflexionar sobre estos temas se busca dar un lugar a esta disciplina, considerando que es fundamental para la transformación de las prácticas de enseñanza y por lo tanto para la intervención social y educativa.

Además, se parte del reconocimiento de la resistencia de los mismos docentes a considerarla como una ciencia y a valorar sus aportes. "La Didáctica no es reconocida como ciencia aun por los propios docentes, muchas veces es relegada y, la mayoría de las veces, diseminada en espacios o parcelas de conocimiento, sin que aún logre las urgentes sistematizaciones provenientes de las conjunciones entre teoría y práctica. Pocos docentes la asumen como una totalidad necesaria para explicar el ser, el saber hacer y el cómo hacerse docente" (Díaz, 1999).

Los autores de este artículo consideran la necesidad de hacer una reconstrucción de su epistemología y comprender en ella probables explicaciones a estas resistencias.

\section{Antecedentes y objeto de estudio de la didáctica}

Las primeras obras que se han encontrado sobre didáctica hacen referencia a las obras del alemán Wolfgang Ratke (1614-1618) y de Comenio (1592-1670). Quizás una de las más conocidas de Comenio sea Didáctica Magna, que es una serie de tratados para la enseñanza de la ciencia, las lenguas, las artes, la piedad y las costumbres.

Es posible que el surgimiento de la pregunta por la enseñanza en la época de Comenio sea consecuencia del contexto moderno que dio prioridad a la educación como forma de culturización y favorecimiento de los ideales de la Ilustración. $\mathrm{Su}$ obra refleja el pensamiento de la época en que la razón imperó sobre otras formas de conocimiento y se validaban sólo aquellos fenómenos que podían ser comprobados experimentalmente. El desarrollo de disciplinas como la fisiología y la psicología fue un aporte a la epistemología de la enseñanza de la ciencia, porque se consideraba necesario que el conocimiento empezara por los sentidos.

La psicología y la fisiología habían explicado los procesos de percepción y memoria en relación con el aprendizaje y la adquisición de conocimientos. 
Así, en la obra de Comenio se encuentran algunas observaciones que dan cuenta del predominio de este pensamiento: "Si se quiere engendrar en los discípulos verdadero y exacto conocimiento de las cosas, hay que procurar que en la enseñanza todo sea por medio de la propia intuición y de la demostración sensual" (Comenio, 1988).

En general, los principios propuestos por Comenio explicitan métodos de enseñanza que, por una parte, favorecen el surgimiento de la razón y, por otra, permiten a todos el acceso al conocimiento. Desde este contexto, la didáctica cobra importancia en cuanto se le asume como una ciencia que establece principios de enseñanza que favorecen el cumplimiento del proyecto educativo moderno. Además, es importante destacar que la educación era principalmente impartida a los niños y los jóvenes; es decir, los métodos de enseñanza estaban dirigidos a los infantes y no a los adultos. Por eso, Comenio afirma:

Oh, qué esperanza tan grande de que se traigan a mejor camino las confesiones humanas que inundan el mundo entero, se aparece ante nuestros ojos, si desde la más tierna edad se acostumbran a todos a ceder ante los demás y a guiarse en todas su cosas por la luz de la razón (Comenio, 1988. p.117).

En consecuencia, la conformación de las explicaciones didácticas va ligada a la enseñanza del infante hasta que alcance su mayoría de edad. Esto influye en la estructura de la ciencia didáctica, porque se asume que su orientación es hacia la enseñanza durante la infancia. Por esta razón, muchos educadores siguen considerándola inapropiada en otros contextos, por ejemplo en el espacio universitario (Díaz, 1995).

Por otra parte la didáctica, así como otras ciencias, recibe aportes de otras disciplinas, siendo la psicología la que hace uno de los mayores aportes. Al revisar los paradigmas psicológicos podrá observarse cómo las diferentes corrientes de esta ciencia influyen directamente en el pensamiento didáctico. El conductismo, por su parte, se ocupa principalmente del estudio del aprendizaje desde una posición causal (estímulo-respuesta). Según esta corriente, el ambiente afecta el comportamiento del individuo, lo que significa que un ambiente específico coadyuva en el aprendizaje, evaluado por el número de aciertos y errores. (De Camilloni et al., 1998).

Esto produce una mirada del estudiante como un ser pasivo frente al conocimiento. Sin embargo el desarrollo de otras disciplinas, por ejemplo matemáticas y física, aporta desde su teoría de campo a otras disciplinas, entre ellas la didáctica, transformando el paradigma causal por una perspectiva interaccionista. Kantor (1924) propone una nueva comprensión del comportamiento desde el plano biopsicosocial, donde lo biológico, lo social, lo psicológico, lo cultural y lo contextual intervienen en la forma como el individuo interactúa con el ambiente. 
El modo como se aborde el proceso del aprendizaje implica un individuo activo y una enseñanza como proceso independiente, aunque en interrelación con el aprendizaje.

Otras investigaciones sobre los estilos de aprendizaje (Gadner, 1995), corrientes constructivistas del conocimiento (Piaget, 1973; Wertsch, 1995), aprendizaje significativo (Ausubel, Novak y Hanesian, 1983) y aprendizaje colaborativo, muestran las tendencias actuales, sin olvidar el papel de la introducción de las nuevas tecnologías en el ámbito educativo. Además el pensamiento filosófico, modernista y postmodernista, y la transformación social y cultural en un mundo globalizado y una sociedad del conocimiento, inciden directamente en la construcción del objeto de estudio de la didáctica actual.

Díaz (1995) explica cómo la investigación psicológica, la extrapolación de conceptos de otras ciencias, el aporte de corrientes filosóficas como el marxismo, el neomarximo y el estructuralismo, el psicoanálisis y la teoría crítica, entre otras, han contribuido al enriquecimiento de la didáctica como teoría de enseñanza. Sin embargo, y a pesar de las críticas a las que ha sido sometida la didáctica, ha venido ocupándose de problemas particulares al interior del aula de clase y con relación al contexto institucional y mundial. Por tanto, se han sumado aportes a la construcción teórica de la didáctica, lo que incide directamente sobre las prácticas de enseñanza.

Es por ello que la didáctica es una ciencia en construcción que determina su propio campo de investigación, sin desconocer que es "heredera y ha sido siembra de otras disciplinas (De Camilloni et al., 1998), siendo esta una característica propia altamente positiva, que no se ha aislado del movimiento general de las ciencias, de cada una de las épocas en que se ha construido un discurso didáctico de relevancia teórica".

Esta construcción de la didáctica en el entorno actual plantea una serie de retos para los investigadores que, a la luz de la teoría educativa crítica, han intentado un acercamiento entre teoría y práctica, por ser posiblemente inseparables. Carr y Kemmis (1988) plantean que la teoría guía el hacer práctico y la práctica aporta a la construcción teórica. La didáctica se convierte así en una ciencia práctica sobre la enseñanza, por lo que se hace necesario revisar, por una parte, el concepto de práctica, y por otra, el de enseñanza, haciendo construcción teórica a partir del análisis de estas prácticas.

La palabra práctica viene del griego praktikos que se refiere a la "acción" y que en el latín toma dos formas, praxis para significar "uso, costumbre" y practice, como el "modo de hacer". La práctica, dicen Campo \& Restrepo (2002), se refiere a los modos observables de operar y que al observarlos nos descubren métodos. Es decir, "diferentes caminos y trayectos implícitos en la acción [...] la práctica hace parte perceptible la forma de acción; su principio organizador 
posibilita la multiplicidad de formas en donde se revelan estilos como el modo distintivo de la Forma, de las formas de actuar [...] las prácticas, múltiples, diversas, inagotables son la expresión de la existencia, donde se cuida el sentido, donde se hace visible aquello que nos distingue y nos une como seres humanos [...] las prácticas responden a una lógica como individuo y como comunidad construyendo cultura".

Por otra parte, la enseñanza es considerada como una tarea compleja que favorece procesos como la humanización, la socialización, la profesionalización y el desarrollo personal (Díaz, 1999). Por lo tanto, la enseñanza en el nivel universitario se constituye como una práctica que debe ser "asumida científicamente y con pertinencia social" (Díaz, 1999). La enseñanza puede verse también como la mediación entre un objeto de conocimiento y un sujeto que se apropia de él.

Así, una teoría sobre las prácticas de enseñanza permite establecer las formas de actuar de los profesores para que el conocimiento, la humanización, la profesionalización y el desarrollo personal se fortalezcan en pro de los estudiantes.

La didáctica puede entonces entenderse como una disciplina que aporta a la comprensión de las formas de actuar de quien enseña, a través de la reflexión crítica, y que revela estilos y modos de hacer. Intenta develar respuestas en cuanto al qué enseñar, cómo enseñar y para qué enseñar. Investiga sobre la relación enseñante-alumno, relación fundamental en la que confluyen todas las prácticas educativas, docentes y de enseñanza, que conducen a la construcción de cultura.

La didáctica es una disciplina que aporta a la comprensión de los procesos que se generan en el aula, pero los transciende al explicar sus relaciones con la institución y el contexto. Tiene relación con la pedagogía en cuanto aporta a la formación del ser y a que, siendo una práctica de enseñanza en el sentido de "praxis", no se desliga del sentido educativo.

Dice Lucio (1989): “Sin la perspectiva globalista e integradora de la visión pedagógica, la didáctica es un instrumento para enseñar mejor, sin preocuparse por el "a quién" [...] el alumno no es una máquina pensante, acumuladora y productora (en el mejor de los casos) de conocimiento. [...] Pero por otro lado, tal vez la tentación más frecuente, sobre todo cuando se atraviesan momentos cruciales de reflexión educativa y de replanteamientos pedagógicos, es olvidarse de la didáctica, del arte (y la ciencia) concreto de la enseñanza".

Es importante reconocer la relación de la didáctica con otras ciencias, aceptar que es heredera de otras disciplinas, que tiene un objeto de estudio que se define, construye y reconstruye día a día en las prácticas cotidianas, que es una disciplina práctica que no se aparta de la teoría, que está recobrando su importancia en el contexto escolar y abriendo caminos en el contexto universitario. 


\section{La didáctica universitaria: teoría de la práctica de enseñanza intencionada y acción de intervención social}

La didáctica universitaria puede considerarse como una didáctica especializada porque su objeto de estudio es el proceso de enseñanza en el ámbito universitario. La construcción teórica sobre lo que acontece en las aulas universitarias y la relación de estos procesos con el espacio institucional y los contextos local y global configuran una serie de características propias de su objeto de estudio.

Desde este punto de partida y comprendiendo que el sentido de las prácticas de enseñanza en el ámbito universitario es diferente, la didáctica asume nuevas posturas frente a los retos que se proponen a la universidad en un mundo globalizado.

La educación universitaria colombiana está pasando por una serie de procesos propios a este fenómeno de la mundialización. La acreditación, la flexibilización, los sistemas de evaluación de la calidad y los estándares impuestos desde afuera revalúan el papel de la universidad y, por tanto, del docente universitario. En general, y debido a los resultados de estos procesos, parece ser que las instituciones universitarias colombianas empiezan a mirar sus procesos internos, y en este sentido esa mirada va también dirigida a los procesos de enseñanza.

Así, la didáctica universitaria es aun más reciente que la didáctica general y asume problemas particulares y específicos; en general, las preguntas que intentan responder están no sólo en relación con la enseñanza sino con la formación del futuro profesional. La didáctica universitaria analiza e investiga la sociedad actual del conocimiento, las tendencias hacia las competencias laborales, el uso de las nuevas tecnologías de información y comunicación y su incidencia en el profesional que se espera formar para el futuro, transformando así el papel de la universidad y en consecuencia el de la didáctica, que se ve abocada a generar nuevas preguntas y respuestas que den cuenta de estos fenómenos sociales y culturales en relación con lo que se presenta en el aula universitaria.

Como puede observarse, la mirada que hace la didáctica responde al contexto macro (social y cultural) y al contexto micro (aula de clase). Así, la didáctica universitaria intenta explicar las interrelaciones universidad-políticas universitarias-comunidad académica-docente-estudiante y no se limita a estudiar un conjunto de métodos (didáctica instrumental) sino que, más bien, presenta posturas críticas frente a sus procesos (didáctica crítica).

La enseñanza universitaria, vista desde esta perspectiva, se configura como una labor compleja que orienta las prácticas del docente en un proceso autorreflexivo y transformador que incide en los procesos de aprendizaje y formación del estudiante. Por tanto, la didáctica universitaria se convierte en una acción de 
intervención social mediada por procesos de enseñanza intencionada, acorde con los cambios que exige la sociedad de hoy y con las proyecciones futuras.

La didáctica es una disciplina que genera conocimiento sobre el proceso intencional de la enseñanza, orientando la praxis y evaluando la actuación de los docentes (Díaz, 1995). En tal sentido es una ciencia de intervención social porque transforma al docente y al individuo en la interrelación que se presenta en el aula.

Desde el análisis crítico, permite desentrañar las ideologías que se encuentran detrás de los procesos curriculares, en el ámbito institucional y en las ideologías de los docentes en el aula de clase. Estas ideologías muestran modos de hacer del docente, que le dan sentido a su hacer y que se hacen desde un estilo particular.

La comprensión de las ideologías que guían la acción permite realizar transformaciones que se orientan hacia la generación de mejores prácticas de enseñanza. Por ello, la tarea de enseñar se convierte en un proceso autorreflexivo, intencional y sistemático, que permite no sólo instruir sino formar seres humanos y que "implica asumir el debate antropológico, ontológico y axiológico en un contexto investigativo particular" (Lucarelli, 2000).

Sin embargo, y como se mencionó al inicio de este escrito, la construcción didáctica en general, y en particular la universitaria, se ve sometida a resistencias por parte de sus principales actores, los docentes. Díaz (1999) explica este fenómeno a partir de cuatro actitudes:

1. "Existen ciertas reticencias del propio profesorado universitario para reconocerla como piso teórico de envergadura y para comprender que para enseñar no basta con saber la asignatura.

2. Persiste un descuido sobre investigación en esta área por parte de quienes tienen la enseñanza por profesión.

3. Poca reflexión y divulgación didáctica en este ámbito.

4. Escaso interés institucional de convertir la enseñanza superior en un verdadero proceso científico".

Parte de las explicaciones sobre estas resistencias tiene su origen, como ya se explicó, en la conformación de la didáctica como ciencia. Sin embargo, posibles explicaciones a estos fenómenos tienen que ver con la "dificultad humana" de observar sus propios procesos, con su complejidad y con el proceso de aceptación y confrontación. En ese aspecto existe un amplio campo de investigación didáctica.

Como consecuencia, las iniciativas investigativas propias de la didáctica universitaria deben ser acordes con las tendencias actuales en relación con: (a) 
procesos de enseñanza que generen aprendizajes significativos; (b) incorporación innovadora en el aula de clase, dentro de la cual las nuevas tecnologías hagan presencia innegable; (c) generación de habilidades metacognitivas; (d) desarrollo personal del estudiante y el docente; (e) lo ético, estético e intelectual; (f) la interdisciplinariedad con otras ciencias como la psicología, la pedagogía, la sociología y las artes; (g) procesos formativos, y (h) procesos curriculares que a su vez den cuenta de los procesos sociales y culturales.

Finalmente, y a manera de conclusión, se puede observar que la didáctica universitaria es a su vez una ciencia en construcción que, como señalan Porlan et al. (1995), debe "cumplir con los desafíos del futuro avanzando en la construcción educativa e interdisciplinar hacia una propuesta crítica de la tarea del aula, que además ilustra sobre la complejidad de los procesos de enseñanzaaprendizaje, y da cuenta de los medios para transformarla" (Díaz, 1995).

Las tendencias actuales y las concepciones acerca de cómo aprenden los individuos y el papel de la enseñanza implican transformaciones e incremento de las investigaciones en este campo. La globalización está exigiendo una formación integral del individuo basada en la generación de competencias que le permitan acceder al mundo globalizado. La necesidad de mayor cobertura y la implementación de cursos virtuales apoyados en las tecnologías de información y comunicación plantean serios cuestionamientos frente al papel de la didáctica.

Algunos de los problemas que deben resolverse tienen que ver con la concepción de lo que es "conocimiento" y su valor. Otro problema está relacionado con la facilidad con que cuentan los estudiantes para acceder a grandes volúmenes de información a través de internet y otros medios, y que permite al individuo estar informado pero no necesariamente generar conocimiento. También están surgiendo nuevas prácticas de enseñanza en las que la mediación de las tecnologías de información y comunicación y el papel del profesor cambian, por lo que es necesario analizar cómo se dan los procesos comunicativos, cómo generar habilidades que integran el manejo de la tecnología con el aprendizaje autónomo, etc.

Así, la enseñanza en un contexto globalizado y a nivel universitario requiere, como dice Díaz (1995), "ser asumida científicamente y con pertinencia social". La intencionalidad de las prácticas de enseñanza orientadas a la formación integral del individuo se constituye así en una forma de intervención social que debe ser asumida con responsabilidad y ética. De las transformaciones favorables que se realicen en el aula, así como de la generación de acciones paralelas, depende en gran parte la formación del futuro profesional. Si no se favorece desde la enseñanza la formación de un pensamiento crítico, posiblemente no será fácil que el estudiante asuma posturas frente a su contexto e incida en él como profesional. 
Es necesario fomentar otras mediaciones y apropiaciones de los contenidos que se pueden enseñar y revisar la calidad de los procesos.

El problema didáctico a nivel universitario también implica el análisis del método y de la evaluación, procesos que, así como la epistemología de la didáctica, también están permeados por otras ciencias, especialmente la psicología. A continuación se realizará un análisis de estos dos aspectos, para comprender mejor el debate actual al interior de la didáctica universitaria.

\section{El problema del método en la didáctica}

En el estudio de los problemas que le competen a la didáctica se asumió durante un tiempo que al resolver el problema del método se podrían resolver todos los problemas didácticos. Sin embargo, el análisis posterior mostró que una visión instrumentalista de la didáctica limitaba su verdadera esencia y papel en la transformación de las prácticas de enseñanza. El debate metodológico desde Comenio hasta los años 1970 y 1980 centró el problema educativo en sus resultados y no en el proceso. El nivel instrumental o tecnicista de la didáctica convirtió al método durante este período en el eje central de la didáctica, haciendo que su objeto fuera simplista y, por tanto, reduccionista.

El problema metodológico se plantea como un modelo de instrucción que se organiza a través de la búsqueda de una técnica que sirva para todos los casos (Díaz, 1995). En los 1990 la preocupación por la calidad de los procesos educativos generó preguntas acerca de qué es conocer, cuál es el conocimiento que se debe adquirir, cómo se debe adquirir ese conocimiento, cómo conoce el ser humano.

En estas condiciones, el problema del método pasa de ser meramente instrumentalista a considerar los entornos y los artefactos que facilitan la apropiación del conocimiento. Según Brown et al. (1989), el método debe estudiar el contexto desde el cual se produce el conocimiento.

Litwin (1997) propone que la manera dinámica como se desarrolla el conocimiento en el aula puede ser vista como una clase que promueve reflexión, o bajo las formas de comunicación que se dan en ella o según la perspectiva moral en la comunicación didáctica de una clase reflexiva.

Díaz (1995) destaca tres categorías para definir el papel del docente frente a la metodología de enseñanza: la ansiedad creadora, la pasión por enseñar y la dimensión intelectual del trabajo docente.

Para Edelstein y Litwin (1993), el problema metodológico implica reconocer la tarea de elaboración del docente como una construcción metodológica que 
deviene en un acto singularmente creativo, que tiene una lógica en relación con lo disciplinar y que permite la apropiación por parte de los sujetos de las situaciones y de los contextos particulares. Además, ha de reconocerse la adopción de una perspectiva axiológica que incide en las formas de vinculación con el conocimiento y que también tiene su expresión en la construcción metodológica.

El problema del método pasa así de ser un "recetario" de enseñanza a una construcción metodológica.

De Camilloni et al. (1998) proponen que para entender la construcción metodológica se deben tener en cuenta por lo menos tres vértices: lo epistemológico objetivo (contenidos, lógicas, etc.), lo epistemológico subjetivo (el conocimiento desde el qué aprender) y lo contextual. El investigador se ubica en el epistemológico objetivo, mientras que el docente se mueve entre lo objetivo y lo subjetivo (transposición didáctica) y es en lo contextual donde las dos lógicas se cruzan.

Para cambiar la categoría de método a construcción metodológica, se tienen que articular los tres vértices de manera creativa, de tal manera que no puede haber homogeneidad ni siquiera al interior de una misma disciplina. Los escenarios son diferentes, los aprendices también; es necesario construir y reconstruir los currículos y los programas que permiten concretar las formas de acción.

Las técnicas de enseñanza no son sino una categoría dentro de la construcción metodológica que ayudan en un determinado momento. Lo metodológico se construye desde la estructura conceptual (sintáctica y semántica de la disciplina), la estructura cognitiva de los sujetos en la situación de aprendizaje, los contextos (institucional, social y cultural) y la perspectiva axiológica.

Como se observa, el problema del método en el debate didáctico es mucho más complejo porque implica la conjugación desde diferentes dimensiones: la del profesor, la del alumno y el contexto. Así, el problema metodológico es específico al interior del aula de clase. El papel del profesor es fundamental porque es quien realiza la intervención didáctica para que el alumno pueda construir significativamente el conocimiento, quien determina la pertinencia de los contenidos y la efectividad del proceso de enseñanza.

El problema didáctico ha de responder a la interacción que hace el maestro en el aula de clase, lo cual implica una construcción metodológica que, desde el vértice subjetivo del maestro, parte de sus propias concepciones acerca de la enseñanza y el aprendizaje. La concepción que tenga el profesor acerca de la forma como sus estudiantes aprenden determinará el "modo" como se enfoca la enseñanza (Porlan et al., 1998). 
Litwin (1997) propone el estudio de las configuraciones didácticas como una forma de analizar las nuevas perspectivas porque permite comprender las tendencias investigativas en este campo disciplinar y la forma como a través de dichos análisis se construyen y reconstruyen nuevos significados en el campo de la didáctica que inciden en la búsqueda de estrategias para mejorar las prácticas de enseñanza.

La investigación didáctica se centra, entonces, en la comprensión de la relación entre enseñanza y aprendizaje, en las configuraciones didácticas de los maestros y en la relación de éstas con la evaluación. Y el problema del método se hace más denso en cuanto no responde a una mirada instrumentalista sino a una más compleja y sistémica.

\section{El problema de la evaluación desde la didáctica}

Considerando el espacio del aula como un escenario de relaciones sociales, la estructura de poder en los centros educativos determina las dinámicas de estas relaciones (Ball, 1993). La figura del profesor, la negociación y la evaluación pueden constituirse en instancias que favorecen el poder del docente en el aula de clase o, por el contrario, están al servicio del aprendizaje y construcción del conocimiento.

La evaluación implica el reconocimiento de los procesos de negociación acerca de las tareas y la vida psicosocial del aula, así como de la comprensión de que "el conocimiento académico no es el único conocimiento escolar existente y posible".

En relación con la evaluación de los aprendizajes, se encuentran algunas contradicciones que están relacionadas con la estrategia que utilizan muchos profesores para controlar el comportamiento del estudiante, que responde en la mayoría de las ocasiones a una calificación, por lo que el sentido educativo que debería tener la evaluación se diluye.

Según Litwin (1997), estas prácticas que tienen por objeto reconocer lo aprendido se convierten en una situación de tensión ajena al ambiente natural en el cual se podría reconocer el aprendizaje adquirido.

Por tanto, en el debate didáctico la evaluación es parte del proceso de enseñanza y aprendizaje, por lo que ha de hacerse un análisis de estos aspectos que abarque posturas éticas y morales de los docentes y actitudes de los estudiantes. Además, los procesos evaluativos corresponden en gran medida a factores externos que exigen a las instituciones responder a estándares que se imponen desde afuera y que no corresponden a las necesidades locales ni tampoco a los procesos del aula en particular. Así que estos factores deben también contemplarse. 
En primera medida vale la pena destacar que la evaluación ha sido utilizada como mecanismo de control y de poder en el ejercicio docente. Se ha asumido como la forma en que los profesores dan cuenta técnica de los aprendizajes de los estudiantes, desconociendo el proceso del aula. Por tanto, está determinada por las concepciones que los profesores tienen acerca de las formas en que el estudiante aprende y de los procesos de enseñanza (Porlan, 1998). Generalmente se ha considerado que un resultado no efectivo en la evaluación, en términos de errores, es consecuencia de ausencia de aprendizaje únicamente, desconociendo si los procesos de enseñanza intervienen en estos resultados.

Según Litwin (1997), el carácter obsesivo por la evaluación da cuenta de una patología del docente frente a la cual el estudiante asume posiciones, convirtiéndose en un hecho aislado del aprendizaje en el que el estudiante busca cómo aprobar y no cómo aprender.

Sin embargo, si se replantea la función de la evaluación, ha de tenerse en cuenta que ésta permite valorar los actos de enseñanza y de aprendizaje. Al respecto Litwin (1997) hace una crítica en cuanto a que el aprendizaje significativo necesita consolidarse en el tiempo y que la evaluación pretende evaluar el aprendizaje como si fuera un acto instantáneo. Desde esta perspectiva, la evaluación debería dar cuenta de la transferencia y generalización de los aprendizajes.

El problema de la transferencia del aprendizaje ha sido estudiado desde perspectivas conductuales y cognitivas, que aunque no siempre tienen el objetivo de aportar a la didáctica sí permiten establecer aspectos que pueden tenerse en cuenta. Ribes, Moreno y Martínez (1998) han venido desarrollando una serie de estudios acerca de la conducta inteligente y el comportamiento verbal, aportando a la comprensión de los procesos de transferencia intramodal y extramodal. En general, sus estudios han mostrado que el uso de instrucciones precisas no permite la abstracción de los elementos fundamentales de un problema o evento y que sólo cuando el individuo se compromete a encontrar las reglas que permiten la solución de un problema y lo resuelve efectivamente, puede observarse transferencia. Sin embargo, estos no son los únicos aspectos que interactúan y permiten la generalización del conocimiento. Ribes (1990) plantea que los estilos interactivos parecen estar relacionados con otros aspectos. Además, se ha encontrando que durante la evaluación de los aprendizajes los individuos pueden aprender, al generar alternativas que implican sus propias construcciones.

La evaluación está demarcada por el comportamiento verbal de los individuos y las acciones comunicativas, lo que implica análisis, síntesis, aplicación de principios y abstracción. Limitar la evaluación a medir únicamente capacidades memorísticas no da cuenta de los verdaderos procesos. También debe permitirse reconocer actitudes y el proceso formativo del estudiante, que son particulares para cada alumno. 
Si se asume que los estilos de aprendizaje median en la construcción del conocimiento, la evaluación no puede ser la misma para todos los estudiantes ya que ha de dar cuenta de sus procesos con relación a su forma de aprender. Desde este punto de vista, el docente debe "entablar el diálogo con sus alumnos para indagar lo que estos desconocen e intentan ocultar" (Litwin, 1997).

Además, ha de crearse en el aula un clima que sea favorable para el desarrollo de estos procesos, desde los cuales el estudiante pueda mostrarse como es, con sus fortalezas y debilidades, y desde donde se atreva a preguntar y cuestionar.

La evaluación debe considerarse, entonces, desde una perspectiva ética y moral, sin descalificar el proceso de los estudiantes. Para ello, el principal desafío desde la didáctica consiste en construir criterios que permitan obtener la información sobre todos los aspectos a evaluarse y que sea válida y confiable. A este respecto, Litwin (1997) dice: "Entendemos que una buena evaluación requiere la formulación y explicitación de antemano de los criterios que se utilizarán para dar cuenta del nivel de la producción, reproducción de información obtenida en clases a partir de lecturas — resolución de un sistema de aplicación — creatividad y originalidad en la respuesta, reconocimiento de niveles diferentes de análisis en lo que respecta a la profundización temática".

\section{La propuesta de Litwin: el estudio de las configuraciones didácticas}

Litwin (1997) considera necesario explicar que la didáctica estudia los procesos comunicativos, los procesos de aprender a enseñar, las prácticas de enseñanza y el estudio de la dimensión en relación con un contexto que les da significado. Esto hace evidente la necesidad de hacer este análisis a la luz de una nueva integración entre los aspectos históricos individuales, las perspectivas y los puntos de vista en relación con los contenidos y métodos y las consecuencias morales del acto de enseñar. Esto implica reconocer la relación que existe entre la construcción del conocimiento y las prácticas de enseñanza.

El conocimiento no se refiere únicamente a la adquisición de información, sino a la generación de habilidad en situaciones concretas, a los procesos cognitivos de aprendizaje y las representaciones del individuo en su interacción con el mundo. El pensamiento del alumno es generador de significados y sitúa al estudiante como transformador activo del conocimiento y constructor de esquemas conceptuales alternativos (Porlan et al., 1998).

Al respecto Driver (1986), al analizar los esquemas conceptuales alternativos de los estudiantes, encontró que estos poseen una coherencia interna que los hace funcionales en determinados contextos experienciales. Esto implica reconocer que 
lo que el estudiante guarda "en su cerebro" tiene sentido en sus construcciones y lo hace responsable de su propio aprendizaje y conocimiento.

Desde esta perspectiva, el conocimiento en el aula y las prácticas de enseñanza para la producción del pensamiento superior implican el análisis de tres aspectos fundamentales: la comunicación didáctica, la perspectiva moral y el análisis de la clase reflexiva. Esta última requiere un análisis de la teoría educativa crítica, que permita comprender la articulación entre teoría y práctica y que genere comprensión sobre el actuar del docente. Una clase reflexiva genera autonomía y pensamiento crítico, principios fundamentales para la comprensión de la acción de enseñanza que propicia a partir de los conocimientos y del pensamiento activo del estudiante. Los abordajes que se hacen de la clase reflexiva pueden ser desde las actuaciones de los docentes para solucionar problemas y la forma como los solucionan, hasta las estrategias para plantear nuevos problemas.

Por otra parte, la comprensión de la comunicación didáctica está enmarcada en la teoría de la acción comunicativa, que integra diferentes aspectos incluido el proceso comunicativo como una acción humana en la que se liga la acción con mecanismos semióticos. Desde aquí se puede analizar el discurso del docente y su interacción de lo que enseña con los procesos comunicativos del estudiante en el aula de clase.

El análisis del discurso del docente permite comprender la forma como las explicaciones del profesor muestran la relación con los campos disciplinarios, las posturas de él frente a dichas explicaciones, sus puntos de vista y su ideología.

La perspectiva moral en la comunicación didáctica se refiere a una reflexión sobre la condición humana en la que la recuperación de la ética y los valores equivalen a pensar críticamente acerca de la justificación de lo que se enseña. Esta reflexión permite generar diálogo que provoque autocuestionamientos y poner en escena actitudes y conductas del docente frente a sus alumnos.

La enseñanza ha de entenderse como un proceso de búsqueda y construcción cooperativa con el estudiante en el que se define el estilo del docente como un conjunto de actitudes y prácticas concretas que abarcan hábitos, gestos, movimientos corporales, expresiones faciales y tonos de voz (Litwin, 1997).

La configuración didáctica se constituye en una forma de describir la enseñanza comprensiva, de explicar la organización y el desarrollo de los procesos reflexivos y de explicitar la manera como se favorecen los procesos de formación de conocimiento y la relación del oficio con los temas en el campo disciplinario. Los análisis de las configuraciones didácticas están orientados a explicar la manera como los docentes abordan los temas, los presupuestos acerca de cómo aprende el estudiante, los estilos como se negocian los significados, la utilización de estrategias metacognitivas y la articulación entre prácticas y teorías. 
La didáctica como disciplina implicaría, entonces, sistematizar estas configuraciones y generar conocimiento a partir de ellas. La propuesta de Litwin (1997) podría acogerse y utilizar este conocimiento para el mejoramiento de las prácticas de enseñanza. Conocimiento y teorías se hacen indispensables en la actualidad, cuando las tecnologías de información y comunicación y los nuevos escenarios (a distancia, semipresenciales y virtuales) involucran a estudiantes y docentes. Por todo lo anterior se hace necesaria la intervención social de la didáctica.

\section{Conclusiones}

La didáctica es una disciplina que se ha venido construyendo paralelamente al desarrollo de otras ciencias y disciplinas como la psicología, la pedagogía, la sociología y la administración, entre otras. En este sentido la epistemología de cada una de ellas, así como la historia y la evolución de estas disciplinas, han tenido impacto directo en la construcción de la didáctica.

Las primeras obras de didáctica tenían una tendencia a explicar métodos didácticos que eran de corte instrumentalista. Esto tenía que ver con la forma en que se entendía el aprendizaje y la alta influencia del conductismo que lo consideraba como resultado de la estimulación externa. Sin embargo, la misma comprensión del proceso de aprendizaje a nivel de la representación del conocimiento y la construcción condujo a que la didáctica tuviera que considerar otros aspectos menos instrumentales.

La didáctica es una disciplina que teoriza y sistematiza el conocimiento de las prácticas de enseñanza. Éstas ocurren en un contexto social y político y están permeadas por él. De tal manera, la didáctica debe estudiar este contexto, la influencia en el aula, las relaciones de poder y las relaciones entre profesor y estudiante. Comprender los hilos que mueven estas relaciones y el proceso educativo es también muy importante.

Existen problemas específicos para la didáctica como son el método y la evaluación. Entender la didáctica como una disciplina que tiene una metodología no significa que se reduzca a un instrumento. Implica desarrollar un conjunto de procedimientos en permanente cambio, para sistematizar las prácticas y, a partir de allí, introducir los cambios. La didáctica entonces se convierte en una disciplina con métodos para la investigación y la intervención en el aula. La evaluación como parte de la práctica de la enseñanza se convierte también en un problema de estudio de la didáctica. La comprensión de estos fenómenos es de mucha importancia para generar cambios al interior del aula. 
Existen diferentes propuestas para comprender la didáctica; una de ellas es estudiar las configuraciones didácticas con base en las interacciones, la comunicación, la ética y la moral. Esta propuesta puede tener relevancia en estudios actuales, ya que las aulas han traspasado el límite físico y se han hecho "virtuales" a través del uso de las tecnologías de información y comunicación.

Es fundamental rescatar la importancia de la didáctica en el mejoramiento de la enseñanza y en el mejoramiento del aprendizaje. El aula, desde esta perspectiva, es un escenario de investigación y el profesor se hace docente-investigador en su propio espacio.

\section{Referencias bibliográficas}

Ausubel-Novak-Hanesian (1983). Psicología Educativa: Un punto de vista cognoscitivo. México: Trillas.

Ball, J. S. Compilador. (1993). Foucault y la educación. Madrid: Narcea.

Brown, J. S., Collins, A. \& Duguid, P. (1989). Situated cognition and the culture of learning. Educational Researcher.

Campo, R., Restrepo, M. (2002). La docencia como práctica. Bogotá.

Carr, W., Kemmis, S. (1988). Teoría crítica de la enseñanza. Barcelona: Martínez Roca.

Comenio, J. (1988). Didáctica Magna. México: Porrúa. Colección Sepan Cuántos. (Trabajo original publicado en 1657).

De Camilloni, A. et al. (1998). Corrientes didácticas contemporáneas. Argentina: Paidós.

Díaz, A. (1995) Didáctica, aportes para una polémica. Rei Argentina: Aique.

Díaz, D. (1999). "La didáctica universitaria. Referencia imprescindible para una enseñanza de calidad: IX congreso de formación del profesorado". Caracas, Venezuela: Revista electrónica Inter. Universitaria de formación del profesorado. En: http://www. emp-virtual.com/datampu/Didactica/didactica.pdf.

Driver, R. (1986). "Psicología cognoscitiva y esquemas conceptuales de los alumnos". Enseñanza de las Ciencias, 4 (1), En: http://www.raco.cat/index.php/ensenanza/ article/viewFile/50854/92858.

Edelstein, G., \& Litwin, E. (1993). Nuevos debates en las estrategias metodológicas del currículum universitario. En Revista Argentina de Educación, AGCE, Año XI, núm. 19, Buenos Aires. 
Gardner, H. (1995). Inteligencias múltiples. Barcelona: Paidós.

Kantor, J. R. (1924-26). Principles of Psychology. New York: Alfred Knopf.

Litwin, E.: (1997). Las configuraciones didácticas, Buenos. Aires: Paidós.

Lucarelli, E. (2000). "El asesor pedagógico en la universidad". En De la teoría pedagógica a la práctica en la formación. Argentina: Paidós-educador.

Lucio, R. (1989) “Educación, pedagogía, enseñanza y didáctica: diferencias y relaciones”. En Revista Universidad de la Salle, núm. 17. Bogotá.

Porlán, R., Azcárate, G., Martín Del Rozo, R., Martín, J. \& Rivero, A. (1998). "Conocimiento profesional deseable y profesores innovadores: fundamentos y principios formativos”. En Investigación en la escuela, pp. 23-38, Sevilla.

Ribes, E. (1990). Problemas conceptuales en el análisis del comportamiento humano. México: Trillas.

Ribes, E., Moreno, D. \& Martínez, C. (1998). Second-order discrimination in humans: The roles of explicit instructions and constructed verbal responding. Behavioral Processes, pp. 1-18.

Piaget, Jean (1973). Epistemología genética. Petrópolis: Vozes.

Wertsch, J. V. (1988). La formación social de la mente. Barcelona: Paidós. 